\title{
Research on GPS Receiver Autonomous Integrity Monitoring Algorithm In the Occurrence of Two-satellite Faults
}

\author{
Er Shen Wang ${ }^{1,2, a}$, Xiao Dong Yue ${ }^{1}$,Tao Pang ${ }^{1}$ and Zhi Xian Zhang ${ }^{1}$ \\ ${ }^{1}$ Shenyang Aerospace University, Shenyang 110136, China \\ ${ }^{2}$ Liaoning General Aviation Key Laboratory, Shenyang 110136,China
}

\begin{abstract}
Reliability is an essential factor for GPS navigation system. Therefore, an integrity monitoring is considered as one of the most important parts for a navigation system. GPS receiver autonomous integrity monitoring (RAIM) technique can detect and isolate fault satellite. Based on particle filter, a novel RAIM method was proposed to detect two-satellite faults of the GPS signal by using hierarchical particle filter. It can deal with any system nonlinear and any noise distributions. Because GNSS measurement noise does not follow the Gaussian distribution perfectly, the particle filter can estimate the posterior distribution more accurately. In order to detect fault, the consistency test statistics is established through cumulative log-likelihood ratio (LLR) between the main and auxiliary particle filters (PFs).Specifically, an approach combining PF with the hierarchical filter is used in the process of two-satellite faults. Through GPS real measurement, the performance of the proposed GPS two-satellite faults detection algorithm was illustrated. Some simulation results are given to evaluate integrity monitoring performance of the algorithm. Validated by the real measurement data, the results show that the proposed algorithm can successfully detect and isolate the faulty satellite in the case of non-Gaussian measurement noise.
\end{abstract}

Keywords: Global positioning system (GPS), Receiver autonomous integrity monitoring (RAIM), Hierarchical Particle filter, Fault detection, Fault isolation

\section{INTRODUCTION}

Integrity of global navigation satellite system (GNSS) is important for safety-critical applications, such as aircraft and missile applications. With the development of GNSS and the increasing requirements for satellite navigation and positioning performance, the integrity monitoring is an inseparable part of GNSS. Integrity monitoring can be able to detect and exclude faults satellite that could cause risks to the accuracy and reliability of GNSS positioning, so that GNSS receivers can operate continuously without any degradation in performance[1]. Because it needs a long time for satellite fault monitoring to alarm through the satellite navigation system itself, usually within 15 minutes to a few hours, that can't meet the demand of air navigation. As a result, to monitor the satellite fault rapidly, namely the receiver autonomous integrity monitoring (RAIM) has been researched a lot. At present, with multiple GNSSs development, there is a need for RAIM to identify multiple outliers. The multiple outliers are more frequent due to the additional affects of non line of sight multipath[2-3]. Therefore, the RAIM needs to be able to detect and exclude multiple biases. It is difficult to detect simultaneous multiple faults using conventional snapshot RAIM algorithms, and therefore various filter algorithms have been studied for reducing the measurement noise level so that GNSS receiver can estimate its position more accurately and reliably[4]. However, for example, Kalman filter presumes that the measurement error follows a Gaussian distribution, the performance can degrade if this assumption is not correct. Because GNSS measurement error does not follow a Gaussian distribution perfectly[5], Kalman filter will use an inaccurate error model that may cause performance degradation. Particle filters have been researched over the last few years as an alternative for solving nonlinear/nonGaussian problems. And, the particle filter for fault detection has been widely used[6-7].

Based on the particle filter, the two-satellite faults detection and isolation algorithm was designed. The new integrity monitoring algorithm for RAIM using hierarchical particle filter was proposed. The proposed algorithm estimates a distribution of a measurement residual from the posterior density and detects large residuals to satisfy a false alarm rate. With a nonGaussian measurement error, the algorithm can estimate the distribution of the state more accurately. The work focused on the effect of a non-Gaussian error distribution of the GPS measurement on the integrity monitoring. The paper is organized as follows. First, a theory of a particle filter is briefly reviewed. Then the general scheme of the approach followed by a hierarchical particle filtering based log likelihood ratio (LLR) approach to fault detection and isolation (FDI) are presented. And the consistency test statistics is derived and established. The next section is a description of the system and measurement equation of GPS receiver. Finally, the GPS receiver autonomous integrity monitoring and its usefulness are presented with numerical simulation and experiment.

\footnotetext{
a Ershen Wang: wanges_2016@126.com
} 


\section{PARTICLE FILTER ALGORITHM}

In this section, the principle of PF algorithm will be given. Particle filter is a method based on sequential monte carlo method and sequential importance sampling (SIS). It has a good filtering effect for non-linear and non-Gaussian system state estimation problem by obtaining sampling from the probability density function (PDF) in the state space. These sampling are called particles. Each of the particles has an assigned weight, and the state variable's distribution can then be approximated by a discrete distribution that depends on each of the particles. The probability assigned to each particle is proportional to the weight. These particles are random samples from the priori PDF. With the increasing of number of particles, a good approximation to the required PDF is effectively provided. Through the system state equation and measurement equation, the collection of sampling for approximating random Bayesian estimation of nonlinear system can be predicted and updated. Gordon, first proposed an algorithm of PF. The algorithm is known as the SIR (sampling importance resampling) [8-9]. At present, the particle filter has been widely used in location tracking, robot localization, signal estimation and detection, speech recognition and enhancement, dynamic fault detection system and satellite navigation[10], and so on. Let's consider the PF dynamic state space model below.

$$
\begin{aligned}
& x_{k}=f\left(x_{k-1}, v_{k-1}\right) \\
& z_{k}=h\left(x_{k}, n_{k}\right)
\end{aligned}
$$

Where $\mathrm{x}_{\mathrm{k}}$ is a state vector, $z_{k}$ is an output measurement vector. $f(\cdot, \cdot)$ and $h(\cdot, \cdot)$ are state transition function and measurement function respectively. $v_{k}$ is the process noise vector independent of current state, and $n_{k}$ is a measurement noise vector independent of states and system noise.

The basic flow of particle filter algorithm can be described as the following steps.

\section{(1).Initialized}

According to the priori probability distribution $\mathrm{p}\left(\mathrm{x}_{0}\right)$, the initial particles $\left\{x_{0}(i)\right\}_{i=1}^{N_{s}}$ from the pdf $\left(\mathrm{x}_{\mathrm{k}-1} \mid \mathrm{Z}_{\mathrm{k}-1}\right)$ are generated, and the weight of the particles is $1 / N_{S}$.

(2).Prediction

Using these particles to generate new samples $\left(x_{k / k-1}^{i}, \mathrm{i}=1, \ldots, \mathrm{N}\right)$, which is approximated the predicted PDF $\mathrm{p}\left(\mathrm{x}_{\mathrm{k}} \mid \mathrm{Z}_{\mathrm{k}-1}\right)$.

Where,

$$
x_{k / k-1}^{i}=f\left(x_{k-1}^{i}, v_{k-1}^{i}\right)
$$

(3). Update

After the measurement $z_{k}$ attained, the weight of each particle at time $\mathrm{k}$ instant is updated. The weights are given by the following equation.

$$
w_{k}^{i}=w_{k-1}^{i} p\left(z_{k} \mid x_{k \mid k-1}^{i}\right)=w_{k-1}^{i} p\left(z_{k}-h\left(x_{k \mid k-1}^{i}\right)\right)
$$

Where, $i=1,2,3, \cdots, N$.The weights are normalized by.

$$
w_{k}^{i}=w_{k}^{i} / \sum_{i=1}^{N} w_{k}^{i}
$$

(4). Resampling

From a set of particles $\left(x_{k / k-1}^{i}, w_{k}^{i}\right)$, according to the value of the importance resampling, a new set of particles $\left(\tilde{x}_{k / k-1}^{i}, \mathrm{i}=1, \ldots, \mathrm{N}\right)$ can be gotten.

(5). Estimation

The set of particles can be used to approximate the posterior PDF, that is $\mathrm{p}\left(\mathrm{x}_{\mathrm{k}} \mid \mathrm{Z}_{\mathrm{k}}\right)$ and the estimated value is as follows.

$$
\hat{x}_{k} \approx \sum_{i=1}^{N_{s}} w_{k}^{i} \tilde{x}_{k / k-1}^{i}
$$

Then, $\mathrm{k}=\mathrm{k}+1$, go to step (2).

\section{HIERARCHICAL PARTICLE FILTER FOR TWO-SATELLITE FAULTS DETECTION}

The problem of fault detection (FD) consists of making the decision on the presence or absence of faults for GPS monitored system, and the problem of fault isolation (FI) consists of deciding the present faulty mode among a number of possible modes. In this paper, a fault detection and isolation (FDI) method is designed for GPS integrity monitoring using hierarchical PF algorithm to detect the consistency of GPS system measurements, and then make the consistency of the test statistic. Finally, the consistency of changes caused by fault compares with the detection threshold to determine the moment of fault and fault satellite. In the algorithm, calculating each time the accumulated LLR function, according to the characteristic of the accumulated LLR function, the characteristic is that under normal circumstances the function curve is smooth with time. When the data fluctuates, it will produce a negative drift before the change and after the change it will produces a positive drift[11]. So the fault detection is to decide a model shift or detecting a jump from the normal model.

This particle filter combining with LLR is used to detect and isolate satellite faults, namely, through PF generating the state estimates, the LLR at each time is calculated. Among the window time, the cumulative LLR is gotten. The consistency is checked. Then the satellite faults are detected and isolated. Therefore, the MAIN PF and auxiliary PF particle normalized weights are calculated in every moment, which is easy to do for PF algorithm[12]. Accumulated LLR can be gotten for consistency test to detect whether there is fault satellite.

The fundamental schemes of RAIM are the same, whether multiple-satellite faults or a single-satellite fault using hierarchical PF based on LLR is achieved to FDI. Thus, given the standard deviation of the pseudo-range errors and false alarm probability, the detection thresholds are the same for both situations. The RAIM method to detect two-satellite faults of the GPS is 
adapted by using hierarchical particle filter based probability test.

1) First, after calculating system state estimation with all $\mathrm{N}$ measurements, the corresponding state estimation with remaining $\mathrm{N}-1$ measurements is calculated. Then LLR consistency checking is evaluated, if it exceeds the detection threshold, fault alarm is set, or no fault.

2) If there is a fault, the corresponding state estimation with remaining $\mathrm{N}-2$ measurements is calculated. Then LLR consistency checking is evaluated, if it exceeds the detection threshold, second fault alarm is set, or no fault.

3) Using the method, after two iterations, the detection of two-satellite faults can be implemented. According to the principle of the method, it can also detect multi- satellite faults.

\section{EXPERIMENT TESTING AND RESULTS ANALYSIS}

The experimental raw measurement data are collected by GPS receiver $\mathrm{N} 220$ (positioning accuracy is 2.5 meters (RMS)), the measurement data including the position information of satellites and the pseudoranges were generated for each epoch for 418 epochs. The user's position outputs at a frequency of $1 \mathrm{~Hz}$. During the period of this collected data, there are six satellites used for PVT solution, the number of GPS satellites is $3,15,18,19,21$, 26 respectively, and the corresponding pseudorange value can be expressed as $Y=\left(y_{1}, y_{2}, y_{3}, y_{4}, y_{5}, y_{6}\right)$. At the same time, another $\mathrm{RCB}-4 \mathrm{H}$ receiver produced by the ublox company is used to monitor whether the satellite is working normally. In order to simulate the fault satellite, the biases were intentionally injects into the pseudorange of two satellites. Here, the $50 \mathrm{~m}$ bias was added to No.19 and No.26 satellites at time $90 \sim 120(\mathrm{k}=90 \sim 120)$. In the simulated experiment, the particle number is chosen as $\mathrm{N}=100$, the calculated decision function of window length is selected as $U=30$, the simulated experimental data measurement noise obeys Gaussian kernel Laplace distribution. Some results of applying the proposed FDI algorithm for GPS integrity monitoring were shown as follows.

In order to conduct fault testing, the bias was added into the pseudoranges measurements. And the detection of anomalies with the proposed FDI method for GPS integrity monitoring was tested. Firstly, by inserting errors into nominal GPS data. In this work, the pseudorange measurements of satellites No.19 and No.26 were modified. Then these modified pseudorange measurements were put back into the FDI system for hierarchical filter. The results of first hierarchical PF are shown as figure 1 and figure 2 .

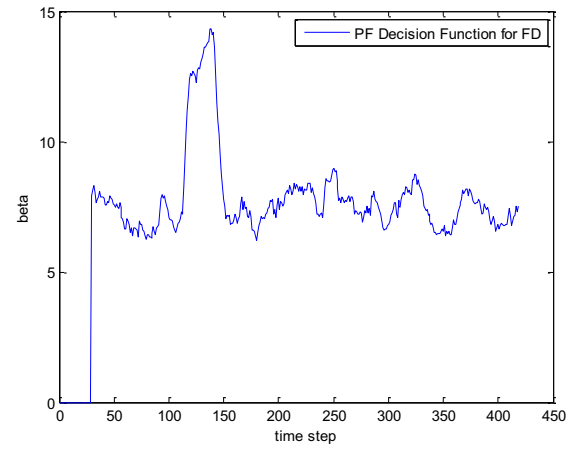

Figure 1. Decision function for fault decision for first hierarchical PF under two-satellite faults condition isolation

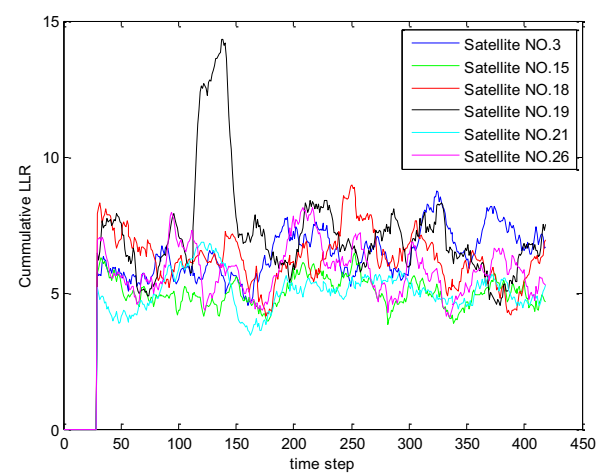

Figure 2. Cumulative LLR for fault for first hierarchical PF under two-satellite faults condition

Figure 1 and Figure 2 show the experimental results of the hierarchical particle filter for GPS RAIM under the first hierarchical PF under two-satellite faults conditions. From figure 1 and figure 2 , it can be seen that the decision function $\beta_{k}$ appeared a significant jump at $\mathrm{k}=95$ that has over the detection threshold. According to the principle of fault detection of the above described, it can be judged that the first satellite No.19 exists fault. When calculating the PVT (position velocity and time) using the data, among the measurement data of the first hierarchical PF, the satellite No.19 should be abandoned. Then the second hierarchical PF continues to detect the other fault satellite. The results of second hierarchical PF are shown as figure 3 and figure 4 .

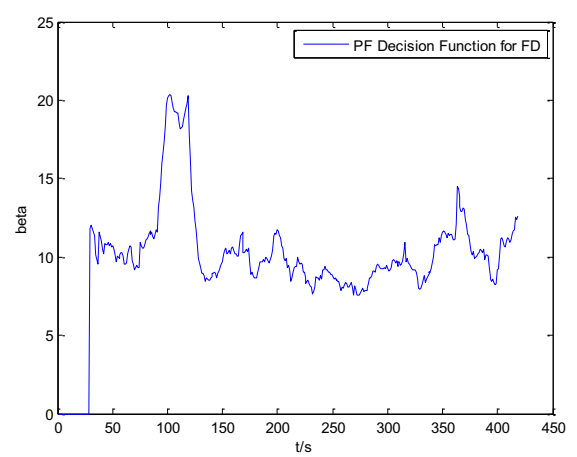

Figure 3. Decision function for fault decision isolation for second hierarchical PF under failure condition 


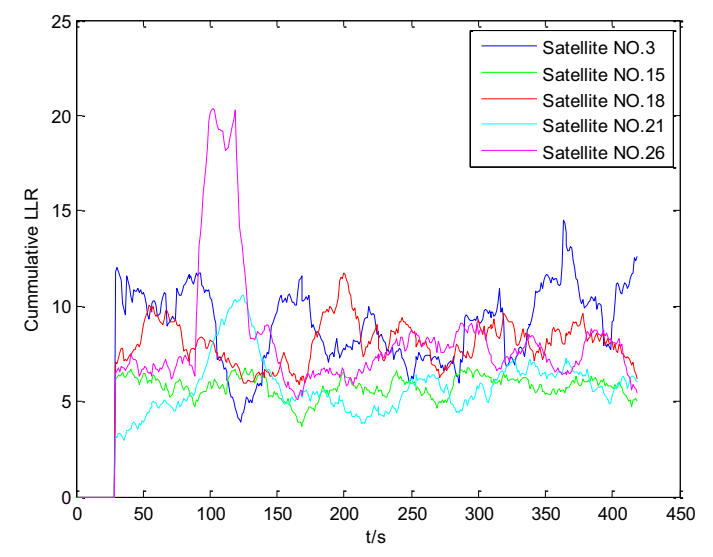

Figure 4. Cumulative LLR for fault for second hierarchical PF under failure condition

Figure 3 and Figure 4 shows the experimental results of the hierarchical particle filter for GPS RAIM under the second of two-satellite faults conditions. From figure 3 and figure 4 , it can be seen that the decision function $\beta_{k}$ appeared a significant jump at $\mathrm{k}=95$ that has over the detection threshold. According to the principle of fault detection of the above described, it can be judged that the satellite No.26 exists fault. When calculating the PVT using the satellite data, the satellite No.26 should be abandoned. So far, two-satellite faults are both abandoned. And the purpose of two-satellite fault detection for GPS integrity monitoring is achieved. The method based on hierarchical PF for GPS RAIM is feasible and effective.

\section{CONCLUSIONS}

A new FDI method for GPS integrity monitoring by using the hierarchical particle filter was proposed. The proposed method makes it possible to detect two-satellite faults for GPS receiver. The hierarchical PF is executed in turn for detecting and isolating two-satellite faults. The test statistics is established .The likelihood function is established and tested by integrating state estimate from both the main PF and auxiliary PFs. Furthermore, the LLR test is used to detect fault, which compares the consistency of the measurement between the main PF and auxiliary PFs. The evaluation of FDI is conducted through simulation using the real GPS measurement data. The measured data from GPS receiver are deliberately contaminated with the bias. Based on the simulation result, the proposed approach demonstrated that it can successfully detect GPS measurement fault under nonGaussian measurement noise, and particularly showed its outstanding performance in the aspects of processing multi-satellite faults. The proposed RAIM algorithm has certain reference value for BeiDou navigation receiver autonomous integrity monitoring.

\section{Acknowledgments}

This research was supported by the National Natural Science Foundation of China (No.61571309,61101161), The Aeronautical Science Foundation of China (No.
2011ZC54010) and the Joint Funds of the Natural Science Foundation of Liaoning Province(2013024003), and Shenyang Aerospace University Doctor start-up fund(15YB23).

\section{References}

1. $\quad$ Ling Yang, Nathan L. Knight, Yong Li, etc, Optimal Fault Detection and Exclusion Applied in GNSS Positioning, JOURNAL OF NAVIGATION, 66, 683-700(2013).

2. JUAN BLANCH,TODD WALTER,PER ENGE,RAIM with Optimal Integrity and Continuity Allocations Under Multiple Failures,IEEE TRANSACTIONS ON AEROSPACE AND ELECTRONIC SYSTEMS, VOL. 46, NO. 3,12351247(2010).

3. J. Liu, M. Lu and Z. Feng, Theoretical analysis of RAIM in the occurrence of simultaneous twosatellite faults,IET Radar Sonar Navig.,1, (2), pp. 92-97(2007).

4. Nathan L. Knight and Jinling Wang,A Comparison of Outlier Detection Procedures and Robust Estimation Methods in GPS Positioning, JOURNAL OF NAVIGATION, 62, 699-709(2009).

5. I.Sayim,B. Pervan, S. Pullen,etc, "Experimental and theoretical results on the LAAS sigma overbound", Proceedings of the ION GPS, Portland, Oregon:2938(2002).

6. YUN Y, KIM D, “Integrity monitoring algorithms using filtering approaches for higher navigation performance:consideration of the non-gaussian gnss measurements" ,Proceedings of ION GNSS 20th International Technical Meeting of the Satellite Division, Fort Worth, TX:3070-3081(2007).

7. Rosihan, Arif Indriyatmoko, Sebum Chun,etc, "Particle Filtering Approach to Fault Detection and Isolation for GPS Integrity Monitoring " , Proceedings of ION GNSS 19th International Technical Meeting of the Satellite Division, Fort Worth, TX:873-881(2007).

8. GORDONN J, ALMOND S, D J, et al,Novel approach to nonlinear/non-Gaussian Bayesian state estimation,IEEE Proceedings F Radar and Signal Processing, 140(2):107-113(1993).

9. M. S. Arulampalam,S.Maskell,N.Gordon,etc, A tutorial on particle filters for online nonlinear/nonGaussian Bayesian tracking,IEEE Transactions on Signal Processing,50(2):174-188(2002).

10. Fredrik Gustafsson, Fredrik Gunnarsson,etc,Particle filters for positioning, navigation, and tracking,IEEE Trans. on Signal Processing,50(2): p 425-437(2002).

11. LI P, KADIRKAMANATHAN V,Particle filtering based likelihood ratio approach to fault diagnosis in nonlinear stochastic systems,IEEE Trans. Syst., Man,Cybern. C, 31(3):337-343(2001).

12. E Kaplan, C Hegarty,Understanding GPS: Principles and Applications,Second Edition. MA, US:Artech House, Inc, 239-264(2006). 